\title{
O CONSUMO DE MACONHA NA ADOLESCÊNCIA E AS CONSEQÜÊNCIAS NAS FUNÇÕES COGNITIVAS
}

\author{
Maisa dos Santos Rigoni \\ Margareth da Silva Oliveira" \\ João Feliz Duarte de Moraes \\ Luis Fernando Zambom ${ }^{\infty}$
}

\begin{abstract}
RESUMO. Este estudo objetiva comparar o desempenho nas funções cognitivas de 30 usuários de maconha e de 30 não usuários. Os instrumentos usados foram uma entrevista estruturada; Screening Cognitivo (WISC-III e WAIS-III); WCST e Figuras Complexas de Rey. A amostra foi de 60 adolescentes, idades entre 14 e 17 anos, e escolaridade mínima de $5^{\text {a }}$ série do Ensino Fundamental. Observou-se que houve diferença estatisticamente significativa no desempenho dos dois grupos em todos os testes neuropsicológicos aplicados; no subteste Código e no WCST, o grupo de usuários denotou desempenho mais prejudicado. Concluiu-se que a maconha pode afetar funções cognitivas de adolescentes usuários.
\end{abstract}

Palavras-chave: adolescência, maconha, testes neuropsicológicos.

\section{THE USE OF CANNABIS IN ADOLESCENCE AND IT'S EFFECTS IN THE COGNITIVE FUNCTIONS}

\begin{abstract}
The objective of the study was to compare the cognitive function performance between a group of 30 cannabis users and a group of 30 non-users. The instruments used were a structured interview; Cognitive Screening (WISC - III and WAIS-III); WCST and Rey Complex Figure Test. The sample was 60 adolescents, aged from 14 to 17 from $5^{\text {th }}$ grade on. The results showed that was statistically significant difference in the performance of the two groups in every neuropsychological test applied, and, in the Digit Symbol and in the WCST subtests, the users' group showed more impaired performance. The findings in this study it can be concluded that cannabis may affect the cognitive functions of adolescents.
\end{abstract}

Key words: Adolescence, cannabis, neuropsychological tests.

\section{EL CONSUMO DE MARIHUANA EN LA ADOLESCENCIA Y SUS CONSECUENCIAS EN LAS FUNCIONES COGNITIVAS}

RESUMEN. Este estudio tiene como objetivo el de comparar el desempeño en las funciones cognitivas de 30 usuarios de marihuana y de 30 no usuarios. Los instrumentos usados fueron una entrevista estructurada; Screening Cognitivo (WISC-III y WAIS-III); WCST y Figuras Complejas de Rey. La muestra fue de 60 adolescentes, con edad entre 14 y 17 años y escolaridad mínima de $5^{\circ}$ año de la educación primaria. Se observó que hubo diferencia estadísticamente significativa en el desempeño de los dos grupos en todos los testes neuropsicológicos aplicados; en el sub-test código y no WCST, el grupo de usuarios obtuvo desempeño más perjudicado. Se concluyó que la marihuana puede afectar funciones cognitivas de adolescentes usuarios.

Palabras-clave: adolescencia, marihuana, testes neuropsicológicos.

* Psicóloga Clínica, Psicanalista. Mestre em Psicologia Clínica pela Pontifícia Universidade Católica do Rio Grande do Sul.

\# Psicóloga, Doutora em Ciências. Professora da Pontifícia Universidade Católica do Rio Grande do Sul.

If Matemático. Estatístico. Doutor em Gerontologia Biomédica. Professor da Pontifícia Universidade Católica do Rio Grande do Sul e da Universidade Federal do Rio Grande do Sul.

x Psicóloga pela Pontifícia Universidade Católica do Rio Grande do Sul. 
A adolescência é uma época da vida que envolve riscos, medos, amadurecimento e instabilidades. Os adolescentes procuram com os pares (amigos, turma, "galera") a dose necessária de aconchego, solidariedade e compreensão, o que faz parte de uma adolescência considerada normal. Nesta etapa, os adolescentes querem ser diferentes dos adultos e, ao mesmo tempo, pertencer a um grupo. Então, é esperado que questionem e duvidem de verdades prontas e rebelem-se, expressando, assim, toda sua energia e criatividade. Mas, esta energia também pode ser canalizada para atividades de risco ou lesivas ao próprio bem-estar. É neste momento que as drogas, lícitas e ilícitas, têm a perversa capacidade de desviar o curso de vida dos jovens, por vezes de maneira irreversível (Pinsky \& Bessa, 2004).

Segundo dados do Centro Brasileiro de Informações sobre Drogas Psicotrópicas - CEBRID (Galduróz, Noto \& Carlini, 1997), a maconha é a droga ilícita mais usada no Brasil entre estudantes do Ensino Médio e Fundamental da rede pública de ensino. No levantamento de 1997, as capitais que apresentaram maior consumo foram Curitiba (11,9\%) e Porto Alegre $(14,4 \%)$. Constatou-se que $7,6 \%$ dos estudantes relataram já ter experimentado maconha uma vez na vida.

O uso de maconha proporciona efeitos prazerosos, como: sensação de relaxamento, cinco sentidos mais aguçados, qualquer coisa torna-se divertida, euforia e aumento de prazer sexual. Já os efeitos que causam desprazer são: ansiedade, pânico, paranóia, diminuição das habilidades mentais - especialmente da atenção e memória - diminuição da capacidade motora, aumento do risco de ocorrerem sintomas psicóticos (Laranjeira, Jungerman \& Dunn, 1998).

O uso crônico da maconha pode levar a déficits de aprendizagem e memória, diminuição progressiva da motivação (isto é, apatia e improdutividade, o que caracteriza a "síndrome amotivacional"), piora de distúrbios preexistentes, bronquites e infertilidade (reduz a quantidade de testosterona). No caso de adolescentes, o déficit cognitivo está relacionado a dificuldades na aprendizagem e repetência escolar (Lemos \& Zaleski, 2004).

Estudos recentes têm demonstrado a existência de uma dependência em relação à maconha. Van den Bree e Pickworth (2005) realizaram um estudo com 13.718 estudantes, entre 11 e 21 anos que já haviam experimentado maconha e encontraram que mais da metade $(55 \%)$ continuaram seu uso 1 ano depois. Fergusson e Horwood (2000) em um estudo longitudinal realizado na Nova Zelândia, que acompanhou 1265 crianças, também constataram que 9\% dos jovens de 21 anos preenchiam critérios diagnósticos, conforme o DSM-IV, para dependência em relação à maconha. Coffey, Carlin, Lynskey, Li e Patton (2003) observaram que, entre os usuários de maconha de 1 ano ou menos, 33,6\% além de continuar usando a maconha após 1 ano, ainda aumentaram a quantidade de uso; e os que usavam maconha há 3 ou mais anos, $61,8 \%$ preenchiam critérios para dependência de maconha.

Pesquisas têm demonstrado que o uso crônico de maconha pode provocar déficits cognitivos, alterações em funções associada direta ou indiretamente ao córtex préfrontal, principalmente quando o uso desta substância ocorreu durante a adolescência (Bolla, Brown, Eldreth, Tate \& Cadet, 2002; Solowij \& cols., 2002). Também foram encontradas alterações neuropsicológicas, especificamente em atenção, memória de curto prazo, funções executivas e funções psicomotoras (Bolla e cols., 2002; Pope, Gruber \& Yurgelun-Todd, 1995; Solowij \& cols, 2002).

Muitos instrumentos são utilizados para medir as funções cognitivas na dependência química. Em um estudo com alcoolistas foram utilizados o Teste das Figuras Complexas de Rey - Forma A (Rey, 1999) e o Screening cognitivo do WAIS (Wechsler Adult Intelligence Scale - Wechsler, 1981) que compreende os subtestes Vocabulário, Cubos e Símbolos Numéricos, verificando-se a presença de prejuízos na capacidade de percepção visual e memória imediata, bem como certos prejuízos na capacidade de análise e síntese, lentificação psicomotora e flexibilidade mental (Oliveira, 2001).

Numa pesquisa que avaliou dependentes de cocaína, foram administrados os testes de Trail Making Test (TMT), Stroop Color Word Test (SCWT), Dígitos Diretos e Indiretos da Escala de Memória WechslerRevisada (WMS-R), Vocabulário, Cubos e Códigos da Escala de Inteligência Wechsler-Revisada, Associação verbal controlada (COWA), teste de nomeação de Boston (BNT), Memória Lógica I e II e Reprodução Visual I e II da WMS-R, Teste de Figuras Complexas de Rey, Teste de Rememoração Seletiva (SRT) e o Wisconsin Card Sorting Test (WCST) versão reduzida com 64 cartas. Este estudo demonstrou déficits de atenção, memória visual, memória verbal, funções executivas, capacidade de aprendizagem e coordenação visuomotora (Cunha, Camargo \& Nicastre, 2001). Nassif e Bertolucci (2003) também avaliaram dependentes de cocaína, através de testes que avaliaram atenção, flexibilidade mental, linguagem, memória e praxias visuoconstrutivas, e os dados sugeriram comprometimento neuropsicológico. 
Na pesquisa realizada por Bolla e cols. (2002) para avaliar os efeitos neuropsicológicos em usuários de maconha, através da administração dos testes Wechsler Adult Intelligence Scale- revised (WAIS-R), Wechsler Memory Scales- revised (WMS-R), Rey Auditory Verbal Learning Test (RAVLT), Rey Osterreith Complex Figure, Symbol Digit Paired Associat Learning Test, Trails A e B, Stroop, Wisconsin Card Sorting Test (WCST) e Judgement Of Line Orientationde, que avaliaram memória visual e verbal, atenção, destreza manual, velocidade psicomotora e funções executivas, constatou-se que o uso grave de maconha (78 a 117 cigarros de maconha por semana, no período de 3 a 15 anos) fez com que persistissem alterações cognitivas relacionadas ao córtex pré-frontal, como a memória, funções executivas e destreza manual, após 28 dias de abstinência.

Em outro estudo para investigar os efeitos cognitivos residuais em usuários graves de maconha, foram administrados os testes vocabulário do WAIS-R (Wechsler Adult Intelligence Test Revised), Stroop Test, Wisconsin Card Sorting Test (WCST), Benton Verbal Fluency Test, Wechsler memory Scale, Clifornia Verbal Learning Test e o Rey-Osterreith Complex Figure Test, para a avaliação das funções cognitivas. Os resultados demonstraram que houve uma redução na função do sistema executivo e atencional compreendido pelo decréscimo na flexibilidade mental e perseveração, bem como, redução na capacidade de aprendizado (Pope \& Yurgelun-Todd, 1996).

No estudo longitudinal com usuários exclusivos de maconha, no qual foi aplicado o WISC para se obter o QI (quociente intelectual) na faixa etária dos 9 aos 12 anos e, posteriormente, na faixa etária dos 17 aos 20 anos, aplicou-se o WAIS para a obtenção do QI; observou-se que o uso recente de maconha esteve relacionado com declínio no nível de QI nos usuários graves de maconha, isto é, nos usuários que fumavam ao menos 5 baseados por semana (Fried, Watkinson, James \& Gray, 2002).

Pope e cols. (2003) analisaram os resultados de testes neuropsicológicos de 122 usuários de longo tempo de maconha; destes, 69 começaram a usar a droga aos 17 anos de idade, ou antes, e apresentaram um desempenho pior nos testes que avaliavam as funções verbais, como QI verbal e a memória de palavras, se comparados aos que começaram a usar a maconha quando adultos, ou mesmo os que usavam apenas algumas vezes.

Os efeitos neuropsicológicos associados ao uso grave ou de longo prazo de maconha têm sido interesse de pesquisadores (Pope, Gruber, Hudson, Huestis \& Yurgelun-Todd, 2001; Solowij e cols., 2002). No entanto, a literatura discute as controvérsias quanto à existência de prováveis resíduos neuropsicológicos, mesmo depois de passado o período de intoxicação aguda da maconha.

Solowij e cols. (2002) encontraram diversos déficits neuropsicológicos em usuários de longo prazo, testados numa mediana de 17 horas depois do último uso informado de maconha, principalmente naqueles testes que avaliaram memória e atenção. Estes resultados persistiram depois do período de intoxicação e foram piores conforme o aumento dos anos de uso regular, confirmando que o uso prolongado desta substância provoca problemas cognitivos, e que estes pioram de acordo com o tempo de uso.

Eldreth, Matochik, Cadet e Bolla (2004) observaram a manutenção de alterações funcionais cerebrais em usuários de maconha com 25 dias de abstinência desta droga, esses dados podem ser corroborados por Fried e cols. (2002) que referem que o THC (tetrahidrocannabinol - elemento psicoativo da maconha) produz redução na execução de tarefas que exijam memória, atenção, tempo de resposta imediata e controle motor durante o período de intoxicação, e que podem permanecer por várias horas

Fergusson, Horwood e Swain-Campbell (2002) encontraram em seu estudo uma associação entre o uso da maconha e maiores taxas de evasão escolar, referindo-se a uma chance 3 vezes maior de abandono da escola aos 16 anos em adolescentes que iniciaram o uso desta substância antes dos 15 anos. Também concluíram que o uso regular de maconha pode ser associado a um aumento no risco de uso de outras drogas ilícitas, a um maior envolvimento em crimes, depressão e comportamentos suicidas. Em outro estudo, também realizado por Fergusson, Horwood e Beautrais (2003) foram detectadas associações entre o uso de maconha e crescentes riscos de adolescentes usuários abandonarem os estudos do Segundo Grau (Ensino Médio no Brasil) e de deixarem a escola sem qualificações, fracassando sua entrada na Universidade ou na obtenção de um grau na mesma.

É cada vez mais preocupante o uso de drogas na adolescência e suas conseqüências em termos cognitivos. Diante do exposto acima, faz-se relevante este estudo, que teve por objetivo analisar o desempenho cognitivo de adolescentes abusadores ou dependentes de substâncias psicoativas (sendo a maconha a principal droga de eleição) quando comparados com adolescentes sem abuso ou dependência de substâncias psicoativas. 
Este estudo procurou verificar: se o abuso ou dependência de maconha causa algum déficit cognitivo em adolescentes abusadores ou dependentes desta substância quando comparados aos adolescentes não usuários; e comparar a capacidade de flexibilização do pensamento de adolescentes abusadores ou dependentes de maconha com os sem abuso ou dependência desta substância.

\section{MÉTODO}

\section{Participantes}

A amostra foi constituída de 60 sujeitos, pareados quanto à idade, sexo e nível socioeconômico, que foram divididos em dois grupos: um grupo de 30 participantes usuários de maconha, e outro de 30 participantes não usuários de maconha. Todos os sujeitos foram do sexo masculino, com escolaridade mínima a $5^{\text {a }}$ série do Ensino Fundamental, na faixa etária de 14 a 17 anos.

O grupo de usuários de maconha foi constituído, intencionalmente, por pacientes encaminhados ao LABICO (Laboratório de Intervenções Cognitivas da PUCRS) para a realização de avaliação das funções cognitivas e acompanhamento psicológico através do modelo de Entrevista Motivacional.

O grupo de não usuários foi composto de adolescentes, oriundos de escolas da rede pública de ensino, que não preenchiam critérios de dependência ou abuso de substâncias psicoativas.

Os critérios de exclusão da pesquisa foram: presença de síndrome de privação grave, com sintomas de abstinência da droga (delírios, alucinações) que pudessem alterar o desempenho nos testes neuropsicológicos, transtornos orgânicos cerebrais e transtornos psiquiátricos severos, conforme entrevista estruturada baseada nos critérios do DSM - IV - TR (American Psychiatric Association, 2002).

\section{Instrumentos}

Foi realizada uma entrevista estruturada, baseada nos critérios do DSM - IV- TR (American Psychiatric Association, 2002), para detectar critérios diagnósticos para o uso e/ou abuso de substâncias psicoativas e comorbidades.

Realizou-se, em parte da amostra, um screening toxicológico para detectar o uso de maconha, com o intuito de comparar os relatos dos adolescentes com os resultados obtidos no exame.

Também foram aplicados os seguintes instrumentos:
- Screening Cognitivo do WISC-III (Wechsler, 1991) para os adolescentes de 14 a 16 anos e o Screening Cognitivo do WAIS-III (Wechsler, 1997/2004) para os adolescentes de 17 anos: compreende os subtestes Vocabulário, Cubos, Código e Dígitos: Para avaliar a inteligência prémórbida, conhecimento semântico, estimulação do ambiente e aprendizagem escolar, formação de conceitos envolvendo análise, síntese e organização viso-motora, capacidade de reprodução e imitação, bem como atenção auditiva e memória imediata (Cunha, 2000).

- Teste Wisconsin de Classificação de Cartas (WCST) (Cunha e cols., 2005): é um teste de avaliação cognitiva que foi criado antes da década de cinqüenta, revisado e ampliado nos últimos anos (Heaton, Chleune, Taley, Kay \& Curtiss, 1993). Ele mede a flexibilidade do pensamento do sujeito para gerar estratégias de solução de problemas, com base no feedback do examinador. Permite examinar a capacidade para estabelecer, manter e modificar categorias mentais.

- Figuras Complexas de Rey (Rey, 1959): foi empregado neste estudo a Figura Complexa de A. Rey (1999) - Forma A, que visa à identificação dos prejuízos na percepção visual e memória imediata.

\section{Procedimentos para coleta de dados:}

O presente estudo foi avaliado e aprovado pelo Comitê de Ética da PUCRS.

Todos os participantes assim como seus pais ou responsáveis que aceitaram participar deste estudo assinaram termos de consentimento livre e esclarecido.

Os participantes do grupo de usuários foram encaminhados por diversas instituições ao LABICO (Laboratório de Intervenções Cognitivas da PUCRS), onde se realizou a avaliação cognitiva, com duração de 3 sessões.

O grupo de não usuários, foi oriundo de escolas da rede pública de Porto Alegre que aceitaram participar do estudo.

Em ambos os grupos, uma equipe de auxiliares de pesquisa, previamente treinada, fazia a coleta de dados. Todos os instrumentos foram aplicados de forma individual.

\section{Análise dos dados}

Os dados foram compilados e analisados através do programa SPSS 11.5. e submetidos à estatística descritiva (média, DP, frequiência) e estatística inferencial, utilizando-se o teste Mann-Whitney e o 
teste Qui-quadrado e teste "t", para comparar os dois grupos.

\section{RESULTADOS}

Dos 60 sujeitos que constituíram a amostra, no que se refere aos dados sociodemográficos, o grupo de usuários teve uma média de idade de $15,96(D P=0,76)$ e o grupo de não usuários teve uma média de idade de 15,63 ( $D P=$ 0,71), de acordo com o Teste $t$, para amostras independentes, não houve diferença estatisticamente significativa nesta variável $(p=0,326)$. Quanto ao nível socioeconômico, através do Teste Qui-quadrado, também não houve uma diferença estatisticamente significativa ( $p=$ 0,602 ) entre os dois grupos. No grupo de usuários, observamos que $46,7 \%$ dos participantes obtinham uma renda mensal de até 1000 Reais e 53,3\% mais de 1000 Reais ao mês, e no grupo de não usuários verificamos que a renda mensal de $40 \%$ dos participantes deste grupo era de até 1000 Reais e $60 \%$ mais de 1000 Reais ao mês.

Pode-se observar que $73,3 \%$ dos participantes do grupo de usuários possuíam o Ensino Fundamental incompleto; e apenas 26,7\% o Ensino Médio incompleto. Já no grupo de não usuários, este quadro se inverteu, encontrando-se 26,7\% participantes com o Ensino Fundamental incompleto e 73,3\% com o Ensino Médio incompleto, apontando, conforme o Teste Quiquadrado, uma diferença estatisticamente significativa $(p \leq 0,001)$ entre os dois grupos.

Quanto à presença de comorbidades, foi possível constatar que, no grupo de usuários, 36,7\% dos participantes não preencheram nenhum critério diagnóstico; $36,7 \%$ preencheram critérios para Transtorno de Conduta; 16,7\% apresentaram critérios de Transtorno de Déficit de Atenção/Hiperatividade; e $10 \%$ preencheram critérios para Transtorno Desafiador de Oposição. Já no grupo de não usuários, 96,7\% dos participantes não preencheram nenhum critério diagnóstico; e 3,3\% preencheram critério para Transtorno de Déficit de Atenção/Hiperatividade.

No grupo de usuários, foi realizado screening de urina em $46,7 \%$ dos participantes, dos quais, $30 \%$ apresentaram exame positivo para uso de maconha, e $16,7 \%$ negativo, confirmando o auto-relato dos participantes. Constatamos com isto que uma parte deste grupo encontrava-se abstinente. No grupo de não usuários, $30 \%$ dos participantes fizeram screening de urina, e o resultado foi negativo para o uso de maconha, também confirmando o auto-relato destes participantes. Não se fez screening de urina em 53,3\% dos participantes do grupo de usuários e em $70 \%$ do grupo de não usuários, pois o auto-relato confirmou em $100 \%$ de concordância os resultados dos participantes que realizaram o screening de urina.

Podemos verificar que no grupo de usuários a freqüência dos participantes que preencheram critérios para dependência em relação ao uso de maconha foi de $56,7 \%(n=17)$. A idade mínima com que este grupo consumiu maconha pela primeira vez foi aos 9 anos, e a idade máxima foi aos 16 anos, sendo a média de idade deste primeiro consumo 13,5 anos. Na Tabela 1 observa-se o consumo semanal do grupo de usuários de maconha.

Tabela 1. Distribuição do Grupo de Usuários Quanto à Quantidade de Consumo Semanal de Maconha

Grupo de Usuários $(n=30)$

\begin{tabular}{lrr} 
Variáveis & $\boldsymbol{n}$ & $\boldsymbol{\%}$ \\
\hline Consumo de até 10 baseados por semana & 12 & $40,0 \%$ \\
Consumo acima de 10 baseados por semana & 18 & $60,0 \%$ \\
\hline
\end{tabular}

Ao compararmos o desempenho do grupo de usuários com o grupo de não usuários quanto aos subtestes do WISC -III e do WAIS-III obtivemos os dados ilustrados na tabela 2:

Tabela 2. Análise Comparativa do Desempenho dos Dois Grupos nas Medidas de Funções Cognitivas

\begin{tabular}{|c|c|c|c|c|c|}
\hline \multirow[b]{2}{*}{ Subtestes } & \multicolumn{2}{|c|}{ Grupo de Usuários $(n=30)$} & \multicolumn{2}{|c|}{ Grupo de Não Usuários $(n=30)$} & \multirow[b]{2}{*}{ t-Test } \\
\hline & Média & $D P$ & Média & $D P$ & \\
\hline Vocabulário & 9,96 & 2,17 & 12,06 & 2,57 & $p<0,001$ \\
\hline Cubos & 10,50 & 3,49 & 12,43 & 2,31 & $p=0,014$ \\
\hline Código & 7,80 & 2,99 & 10,63 & 2,69 & $p<0,001$ \\
\hline Dígitos & 8,76 & 2,82 & 12,90 & 4,13 & $p<0,001$ \\
\hline
\end{tabular}

De acordo com os dados da tabela 2, através do Teste t, constatamos que houve uma diferença estatisticamente significativa quanto ao desempenho dos participantes dos dois grupos nos quatro subtestes do WISC-III e do WAIS-III aplicados, mostrando que as médias de desempenho do grupo de não usuários foram mais altas do que do grupo de usuários.

Para avaliarmos a capacidade de percepção visual, medida através do Teste Figuras Complexas de Rey, utilizamos o teste não paramétrico de Mann-Whitney, no qual se obteve uma diferença estatisticamente significativa $(p=0,047)$ entre os dois grupos. Pudemos verificar uma média de desempenho de 26,12 pontos para o grupo de usuários e 34,88 pontos para o grupo de não usuários.

Ao comparmos o desempenho dos dois grupos no que tange à capacidade de memória imediata, avaliada pelo Teste Figuras Complexas de Rey, observamos que a média do grupo de usuários foi de 21,05 pontos e do grupo de não usuários foi de 23,95 pontos, o que, conforme o Teste t, 
aponta para uma diferença estatisticamente significativa $(p=0,047)$.

$\mathrm{Na}$ tabela 3, verificamos o desempenho dos dois grupos quanto à capacidade de flexibilidade do pensamento para gerar estratégias de solução de problemas, avaliada pelo Teste Wisconsin de Classificação de Cartas (WCST):

Tabela 3. Desempenho dos Dois Grupos no WCST

\begin{tabular}{|c|c|c|c|c|c|c|}
\hline \multirow[b]{2}{*}{ WCST } & \multicolumn{3}{|c|}{ Grupo de Usuários $(I=30)$} & \multicolumn{3}{|c|}{ Grupo de Não Usuários $(I=30)$} \\
\hline & Média & Mediana & $D P$ & Média & Mediana & DP \\
\hline Categorias Completadas & 4,8 & 5,00 & 1,29 & 5,6 & 6,00 & 1,02 \\
\hline $\mathrm{N}^{\mathrm{o}}$ Total de Erros & 36,06 & 41,00 & 18,23 & 17,90 & 12,00 & 17,22 \\
\hline Erros Perseverativos & 18,40 & 18,60 & 10,08 & 8,60 & 6,00 & 4,48 \\
\hline Erros Não-perseverativos & 17,33 & 17,00 & 10,61 & 9,3 & 5,50 & 9,99 \\
\hline Ensaios para completar a $1^{\text {a }}$ Categoria & 18,13 & 13,00 & 9,75 & 11,86 & 11,00 & 3,85 \\
\hline $\begin{array}{l}\text { Percentual de Respostas de Nível } \\
\text { Conceitual }\end{array}$ & 60,95 & 58,20 & 16,61 & 77,61 & 82,45 & 16,59 \\
\hline Fracasso em Manter o Contexto & 1,06 & 1,0 & 0,78 & 0,33 & 0,00 & 0,71 \\
\hline Aprendendo a Aprender & $-0,65$ & $-1,22$ & 7,10 & $-2,04$ & $-1,50$ & 6,27 \\
\hline
\end{tabular}

No entanto, observou-se que estas variáveis avaliadas pelo WCST não estavam distribuídas segundo uma curva normal de probabilidade, e, por isso, para compararmos os dois grupos, optamos pelo Teste Mann-Whitney, conforme nos mostra a tabela 4.

Tabela 4. Comparação dos Dois Grupos Quanto às Categorias Avaliadas pelo WCST

\begin{tabular}{lrl}
\hline \multicolumn{1}{c}{ WCST } & Mann-Whitney & $p^{*}$ \\
\hline Categorias Completadas & $-3,20$ & $\leq 0,001$ \\
$\mathrm{~N}^{\circ}$ Total de Erros & $-4,01$ & $\leq 0,001$ \\
Erros Perseverativos & $-4,26$ & $\leq 0,001$ \\
Erros Não-perseverativos & $-3,82$ & $\leq 0,001$ \\
Ensaios para completar a 1 ${ }^{\text {a Categoria }}$ & $-3,98$ & $\leq 0,001$ \\
Percentual de Respostas de Nível Conceitual & $-3,97$ & $\leq 0,001$ \\
Fracasso em Manter o Contexto & $-3,77$ & $\leq 0,001$ \\
Aprendendo a Aprender & $-0,37$ & $=0,711$ \\
\hline
\end{tabular}

* Os resultados foram significativos ao nível de $5 \%$.

Ainda na tabela 4 , podemos verificar que, em todas as categorias do WCST, houve uma diferença estatisticamente significativa entre os dois grupos, exceto na categoria Aprendendo a Aprender, que não apresentou uma diferença estatisticamente significativa $(p=0,711)$.

\section{DISCUSSÃO}

Neste estudo, avaliaram-se adolescentes abusadores e dependentes de maconha, comparados aos adolescentes não usuários de substâncias psicoativas quanto ao desempenho de suas funções cognitivas. Os dois grupos foram pareados pelo sexo, idades e nível socioeconômico, a fim de que se pudesse realizar uma comparação homogênea entre os dois grupos.
Em primeiro lugar, constatou-se que a maioria dos adolescentes do grupo de usuários de maconha possuía o Ensino Fundamental Incompleto, seja por evasão escolar ou mesmo por diversas repetências, enquanto a maioria dos adolescentes do grupo de não usuários possuía o Ensino Fundamental Completo e estava cursando o Ensino Médio. Também verificamos que o grupo de usuários iniciou o consumo de maconha em média aos 13,5 anos. Este dado corrobora os achados de Fergussson e cols. (2002) que encontraram uma associação com maiores taxas de evasão escolar em adolescentes usuários de maconha e que tenham iniciado este uso antes dos 15 anos. Em outro estudo, Fergusson e cols. (2003) também associaram o uso de maconha com crescentes riscos de adolescentes usuários abandonarem os estudos do Ensino Médio e de deixarem a escola sem qualificações, fracassando sua entrada na Universidade ou na obtenção de um grau na mesma.

Em nosso estudo, foram realizados screening toxicológico para o uso de maconha em parte da amostra, no qual se evidenciamos $100 \%$ de concordância entre o auto-relato e o resultado do exame. Este dado corrobora os estudos de Souza e Oliveira (2005), que realizaram em todos os adolescentes de sua amostra o exame de screening toxicológico e obtiveram $100 \%$ de confiabilidade entre o auto-relato e os resultados detectados.

Cabe salientar a prevalência nessa amostra de usuários de maconha de comorbidade com o Transtorno de Conduta (39,7\%). Isto reforça o que Myers, Stewart e Brown (1998) referem em um estudo longitudinal com 137 adolescentes usuários de drogas: entre os meninos, é comum que o uso de drogas seja precedido pelo Transtorno de Conduta. 
Assim como nos estudos de Van den Bree e Pickworth (2005), Fergusson e Horwood (2000), Budney, Hughes, Moore e Novy (2001) e Coffey e cols. (2003), que em seus resultados de pesquisa apontam para a existência de uma dependência em relação ao uso de maconha, em nosso estudo identificamos que mais da metade dos participantes usuários de maconha preencheram critério para dependência desta substância, e, se utilizarmos o critério de Fried e cols. (2002), que consideraram um uso grave de maconha um consumo de mais de 5 baseados por semana, estamos diante de uma população de usuários graves de maconha, pois o consumo destes participantes (60\% da amostra) era acima de 10 baseados por semana. Já para Bolla e cols. (2002), este consumo é considerado leve.

Encontramos diferenças estatisticamente significativa no desempenho dos dois grupos quanto aos subtestes do WISC-III e do WAIS-III, demonstrando que as médias de desempenho do grupo de não usuários foram mais altas do que a do grupo de usuários. Através do desempenho no subteste Vocabulário, os dois grupos apontam um funcionamento intelectual pré-mórbido dentro de limites médios; no subteste de Cubos, os dois grupos também apresentaram escores dentro da média, não sugerindo prejuízos na capacidade de análise e síntese, assim como no subteste Dígitos, no qual, os grupos apresentaram escores dentro de um termo médio, não revelando prejuízos na atenção auditiva e memória imediata. No entanto, no subteste Código, identificamos que o grupo de usuários denota desempenho mais prejudicado com relação à lentificação psicomotora e flexibilidade mental. Estes achados corroboram os resultados encontrados por Pope e cols. (1995), que detectaram a existência de déficits em tarefas psicomotoras, atenção e memória de curto prazo em sua amostra. Laranjeira e cols. (1998) também apontam que o uso de maconha propicia uma diminuição das habilidades mentais especialmente da atenção, memória e diminuição da capacidade motora.

No que tange à capacidade de percepção visual, que envolve atenção, avaliada pela cópia do Teste de Figuras Complexas de Rey, percebemos que o grupo de usuários apresenta prejuízos cognitivos, enquanto o grupo de não usuários denota um desempenho dentro da média. Este dado corrobora os estudos de Pope e cols. (1995), Solowij e cols. (2002) e Bolla e cols. (2002), que também detectaram em suas pesquisas a presença de déficits relacionados à atenção em usuários de maconha.
Quanto à capacidade de memória imediata, avaliada pela reprodução de memória do teste de Figuras Complexas de Rey, verificamos que, apesar de haver uma diferença estaticamente significativa no desempenho entre os dois grupos, seus escores situamse dentro de um termo médio, não sugerindo prejuízos nesta função. Este achado contraria os resultados de outros estudos que encontraram déficits em memória de curto prazo de usuários de maconha (Bolla e cols., 2002; Hall \& Solowij, 1998; Pope e cols., 1995; Solowij e cols., 2002). Talvez por se tratar de uma amostra de adolescentes jovens com um uso recente da maconha.

Através dos escores alcançados nas categorias do WCST, podemos perceber que o grupo de usuários completou menos categorias, cometeu mais erros, perseverou mais erros, necessitou mais ensaios para completar a $1^{\text {a }}$ categoria, obteve um percentual de respostas de nível conceitual mais baixo e fracassou mais para manter o contexto, do que o grupo de não usuários, sugerindo um desempenho mais prejudicado no que se refere às funções executivas requeridas por este teste. Os usuários de maconha demonstraram um desempenho mais prejudicado no que se refere as funções executivas. Os dados referidos corroboram as investigações de Cunha e cols. (2001), que também obtiveram desempenho prejudicado em usuários de cocaína. Bolla e cols. (2002) e Pope e cols. (1996) também encontraram prejuízo em funções executivas de usuários de maconha. No entanto, Solowij e cols. (2002) não encontraram em seus estudos, diferenças estatisticamente significativa nas categorias do WCST, mas sim uma tendência no grupo de usuários de longo prazo de maconha, que falharam para manter o controle (organização) mais freqüentemente do que os usuários de curta duração e do que o grupo de não usuários.

\section{CONSIDERAÇÕES FINAIS}

Diante dos resultados obtidos neste estudo, com esta amostra, pode-se constatar que os adolescentes usuários de maconha apresentaram um desempenho inferior, no que tange as funções cognitivas, quando comparados ao grupo de adolescentes não usuários de maconha, sugerindo que a maconha pode afetar o funcionamento neuropsicológico de usuários.

Evidenciaram-se poucos estudos na literatura com esta faixa etária; muitas pesquisas encontradas objetivaram analisar uma faixa etária mais ampla. Também há muita controvérsia quanto ao que é considerado um usuário grave ou, mesmo, um usuário 
de longo prazo de maconha, o que dificulta classificarmos a amostra pesquisada neste estudo.

A escassez de estudos neuropsicológicos envolvendo o uso de maconha reforça a necessidade de ampliação de estudos com amostra maiores, bem como estudos longitudinais que possam avaliar constantemente os efeitos provocados pela maconha

Neste estudo nos deparamos com limitações importantes, como a faixa etária avaliada (14 a 17 anos), que é restrita e encontra-se muitas vezes no início do uso de maconha, não sendo percebidos, portanto, ainda prejuízos em suas capacidades cognitivas e por isso não chegando aos serviços de atendimento especializados.

Outro ponto relevante é que a amostra aqui estudada ficou restrita, devido ao tempo destinado para a coleta de dados da dissertação de mestrado. Sugere-se a realização de estudos com amostras maiores e com uma faixa etária ampliada.

\section{REFERÊNCIAS}

American Psychiatric Association (2002, 4ª Ed.). Manual diagnóstico e estatístico dos transtornos mentais - DSM- IV TR. Porto Alegre: Artes Médicas.

Bolla, K. ., Brown, K., Eldreth, D., Tate, K. \& Cadet, J. L. (2002). Dose-related neurocognitive effects of marijuana use. Neurology, 59, 1337-1343.

Budney, A. J., Hughes, J. R., Moore, B. \& Novy, P. L. (2001). Marijuana abstinence effects in marijuana smokers maintained in their home environment. Archives of General Psychiatry, 58(10), 917-924.

Coffey, C., Carlin, J. B., Lynskey, M., Li, N. \& Patton, G. C. (2003). Adolescent precursors of cannabis dependence: Findings from the Victorian Adolescent Health Cohort Study. British Journal of Psychiatry, 18,330-6.

Cunha, J. (2000). Psicodiagnóstico: V. Porto Alegre: Artes Médicas.

Cunha, J., Trentini, C. M., Argimon, I. I. L., Oliveira, M. S., Werlang, B. S. G. \& Prieb, R. (2005). Teste Wisconsin de classificação de cartas: manual. São Paulo: Casa do Psicólogo.

Cunha, P., Camargo, C. H. P. \& Nicastri, S. (2001). Déficits neuropsicológicos e cocaína: um estudo-piloto. Jornal Brasileiro de Dependência Química, 1(1), 31-7.

Eldreth, D. A., Matochik, J. A., Cadet, J. L. \& Bolla, K. I. (2004). Abnormal brain activity in prefrontal brain regions in abstinent marijuana users. NeuroImage, 23, 914-920.

Fergusson, D. M. \& Horwood, L. J. (2000). Cannabis use and dependence in a New Zealand birth cohort. New Zealand Medical Journal, 113, 156-158.

Fergusson, D. M., Horwood, L. J. \& Swain-Campbell, N. (2002). Cannabis use and psychosocial adjustment in adolescence and young adulthood. Addiction, 97(9), 1123-35.

Fergusson, D. M., Horwood, L. J. \& Beautrais, A. (2003). Cannabis and educational achievement. Addiction, 98, 168192.
Fried, P., Watkinson, B., James, D. \& Gray, R. (2002). Current and former marijuana use: Ppreliminary findings of a longitudinal study of effects on IQ in young adults. Canadian Medical Association Journal, 166(7), 887-91.

Galduróz, J. C. F., Noto, A. R. \& Carlini, E. A. (1997). IV Levantamento sobre o uso de drogas entre estudantes de $1^{\circ}$ e $2^{\circ}$ graus em 10 capitais brasileiras - 1997. Centro Brasileiro de Informações sobre Drogas Psicotrópicas. São Paulo: UNIFESP.

Hall, W. \& Solowij, N. (1998). Adverse effects of cannabis. The Lancet, 352(14), 1611-16.

Heaton, R. K., Chleune, G. J., Taley, J. L., Kay, G. G. \& Curtiss, G. (1993). Wisconsin card sorting test manual (Revised and expanded). Odessa: Psychological Assessment Resources.

Laranjeira, R., Jungerman, F. S. \& Dunn, J. (1998). Drogas: maconha, cocaína e crack. São Paulo: Contexto.

Lemos , T. \& Zaleski, M. (2004). As principias drogas: como elas agem e quais os seus efeitos. Em I. Pinsky \& M. Bessa, Adolescência e drogas (pp. 16-29). São Paulo: Contexto.

Myers, M. G., Stewart, D. G. \& Brown, S. A. (1998). Progression from conduct dosorder to antisocial personality disorder following treatment for adolescent substance abuse. American Journal Psychiatry, 155, 479-485.

Nassif, S. L. S. \& Bertolucci, P. H. F. (2003). Aspectos neuropsicológicos na dependência química: cocaína: um estudo comparativo entre usuários e controles. Em S. L. S. Nassif \& P. H. F Bertolucci, Cérebro, inteligência e vínculo emocional na dependência de drogas (pp.85-105). São Paulo: Vetor.

Oliveira, M. S. (2001) Eficácia da intervenção motivacional em dependentes do alcohol. Tese de Doutorado não publicada, Universidade Federal de São Paulo/Escola Paulista de Medicina, São Paulo.

Pinsky, I. \& Bessa, M. (2004) Adolescência e drogas. São Paulo: Contexto.

Pope, H. G., Gruber, A. J. \& Yurgelun-Todd, D. (1995). The residual neuropsychological effects of cannabis. Drug Alcohol Dependence, 38, 25-34.

Pope, H. G. \& Yurgelun-Todd, D. (1996). The residual cognitive effects of heavy marijuana use in college students. The Journal of the American Medical Association, 275, 521-527.

Pope, H. G., Gruber, A. J., Hudson, J. I., Huestis, M. A. \& Yurgelun-Todd, D. (2001). Neuropsychological performance in long-term cannabis users. Archives of General Psychiatry, 58(10), 909-915.

Pope, H. G., Gruber, A. J., Hudson, J. I., Cohane, G., Huestis, M. A. \& Yurgelun-Todd, D. (2003). Early-onset cannabis use and cognitive deficits: what is the nature of the association? Drug and Alcohol Dependence, 69, 303-310.

Rey, A. (1959). Test de copie d'une figure complexe: Manuel. Paris: Centre de Psychologie Appliquée.

Rey, A. (1999, $1^{\text {a }}$ Ed.). Teste de cópia e reprodução de memória de figuras geométricas complexas: manual. (Margareth da Silva Oliveira, Trad.). São Paulo: Casa do Psicólogo.

Solowij, N., Stephens, R. S., Roffman, R. A., Babor, T., Kadden, R., Miller, M., Christiansen, K., McRee, B. \& Vendetti, J. (2002). Cognitive functioning of long-term heavy cannabis users seeking treatment. The Journal of the American Medical Association, 287(9), 1123-1131. 
Souza, C. C. \& Oliveira, M. S. (2005). Transtorno de déficit de atenção/hiperatividade em adolescentes usuários de drogas. Arquivos Brasileiros de Psiquiatria, Neurologia e Medicina Legal, 99(03), 10-17.

Van den Bree, M. B. \& Pickworth, B. W. (2005). Risk factors predicting changes in marijuana involvement in teenagers. Archives of General Psychistry, 62(3), 311-9.

Wechsler, D. (1991, $1^{\text {a }}$ Ed.). WISC III: Escala de Inteligência Wechsler para Crianças - terceira edição. (Vera Lúcia Marques de Figueredo, Trad.) São Paulo: Casa do Psicólogo.
Wechsler, D. (2004, $1^{\text {a }}$ Ed.). WAIS III- Escala de inteligência para adultos: manual. (Elizabeth Nascimento, Trad.). São Paulo: Casa do Psicólogo. (Original publicado em 1997).

Recebido em 24/02/2006 Aceito em 26/10/2006

Endereço para correspondência: Margareth da Silva Oliveira. Programa de Pós Graduação em Psicologia (PUCRS), Av. Ipiranga, 6681, prédio 11, $9^{\circ}$ andar, sala 932, CEP 90619-900, Porto Alegre- RS. E-mail: marga@pucrs.br 